\title{
RETRACTED ARTICLE: The docking guidance control of underwater glider with integration control algorithm
}

\author{
Zhi-qiang Su${ }^{1} \cdot$ Da-lei Song ${ }^{1} \cdot$ Yu-hai Liu $^{2}$
}

Received: 28 January 2017/ Accepted: 11 June 2017/Published online: 10 July 2017

(c) JASNAOE 2017

The Editor in Chief and the publisher retract this article per COPE guidelines on plagiarism. Shortly after publishing, it was discovered the paper largely plagiarizes the following paper: C. Yang et al. Study on docking guidance algorithm for hybrid underwater glider in currents Ocean Engineering
(2017) 125:170-181, DOI 10.1016/j.oceaneng.2016.08. 002. The authors apologize to the editors and readers as well as the authors of the original paper. The online version of this article contains the full text of the retracted article as electronic supplementary material.

Electronic supplementary material The online version of this article (doi:10.1007/s00773-017-0462-x) contains supplementary material, which is available to authorized users.

Yu-hai Liu

yuhailiu@qq.com

1 College of Engineering, Ocean University of China, No. 238, Songling Road, Laoshan District, Qingdao, Shandong, China

2 College of Information Science and Engineering, Ocean University of China, No. 238, Songling Road, Laoshan District, Qingdao, Shandong, China 